\title{
Multi-wavelength flare study and magnetic configuration
}

\author{
Brigitte Schmieder $^{1,2}$, A. Berlicki ${ }^{1,3}$, N. Vilmer ${ }^{1}$, G. Aulanier ${ }^{1}$, \\ P. Démoulin ${ }^{1}$, P. Mein ${ }^{1}$, C. Mandrini ${ }^{4}$ and E. DeLuca ${ }^{5}$ \\ ${ }^{1}$ Observatoire de Paris-Meudon, LESIA, France email: brigitte.schmieder@obspm.fr \\ ${ }^{2}$ ITA, P.O. Box 1029, Blindern, N-0315 Oslo, Norway \\ ${ }^{3}$ Astronomical Institute of the Wrocław University, Wrocław, Poland, \\ ${ }^{4}$ IAFE, Buenos Aires, Argentina, email:mandini@iafe.uba.ar \\ ${ }^{5}$ Smithsonian Astrophysical Observatory, Cambridge, USA
}

\begin{abstract}
Recent results of two observation campaigns (October 2002 and October 2003) are presented with the objective of understanding the onset of flares and CMEs. The magnetic field was observed with THEMIS and MDI, the chromosphere with the MSDP operating on the German telescope VTT and on THEMIS, the EUV images with SOHO/CDS and TRACE, the $\mathrm{X}$-ray with RHESSI. We show how important is the magnetic configuration of the active region to produce CMEs using two examples: the October 282003 X 17 flare and the October 222002 M 1.1 flare. The X 17 flare gave a halo CME while the M 1.1 flare has no corresponding CME. The magnetic topology analysis of the active regions is processed with a linear-force-free field configuration.
\end{abstract}

\section{October 282003}

The X 17 flare is a complex flare with different phases of evolution inside the quadrupolar magnetic configuration of the active region NOAA 10486 (polarities 1, 2, 3, 4).

Two pre-events are important to explain the major flare (X17) at 11:01 UT (Figure 1). Emergence of small dipoles and converging motions of small polarities towards the main magnetic inversion line create a strong magnetic field stress. The first pre-event is due to the emergence of a small dipole between the main polarities. Small scale reconnection occurs at 10:05 UT and flaring loops last for long time, even after the main flare. The second pre-event signatures are four ribbons, clearly visible in $\mathrm{H} \alpha$ and in TRACE $1600 \AA$. These ribbons could correspond to large scale magnetic reconnection in the quadrupolaractive region. The coronal evolution as seen by TRACE in $195 \AA$, together with TRACE observations in $1600 \AA$ (low chromosphere), indicates that the main X17 flare could be due to the destabilization of a highly twisted flux tube Two main spots ( 3 and 4 ) were at their decay phase with one surrounded by a "moat region". The convergent motions, as the flux diffused towards the main magnetic inversion line, increased the shear and favoured reconnection between the loops in the sheared arcade. This reconnection process, which is visible with the formation of newly reconnected loops before the flare, increased the flux in the twisted flux tube until it finally became unstable and erupted giving raise to a classical two ribbon flare. This phenomena is possible after the large-scale quadrupolar reconnection occurring just before the flare. 

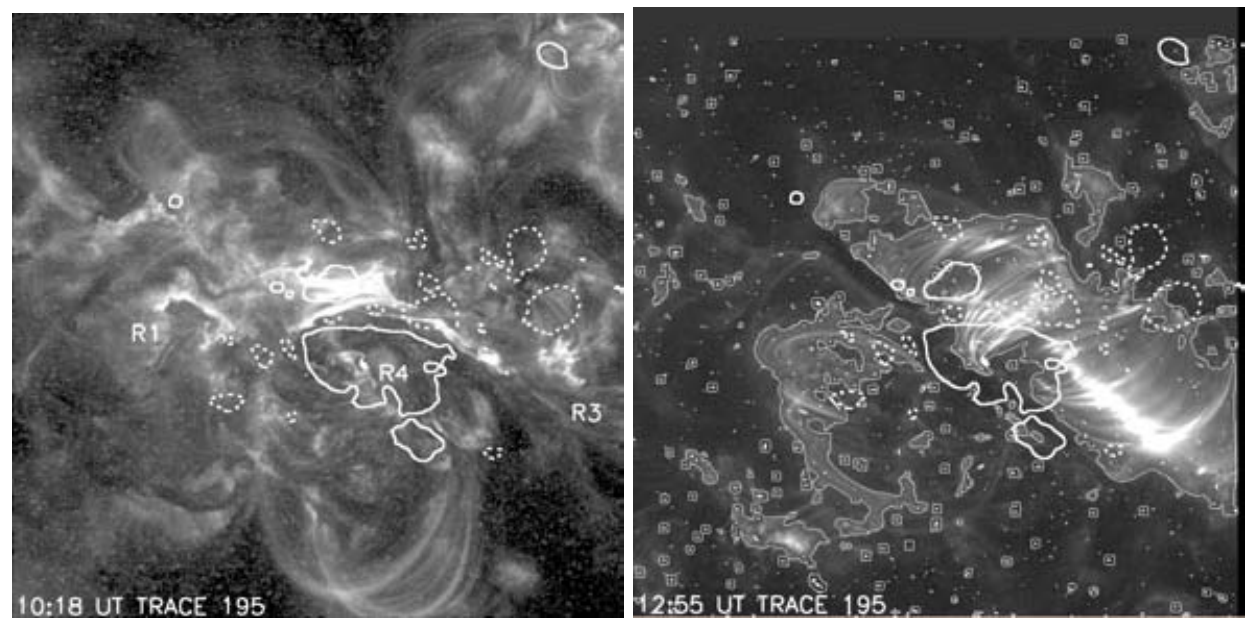

Figure 1. October 28, 2003 , active region NOAA10486 observed in TRACE $195 \AA$ : (left panel) pre-event 1 with compact bright loops and pre-event 2 with four bright ribbons over the main four polarities $(1,2,3,4)$, (right panel) X 17 flare. The images are overlaid by MDI contours.
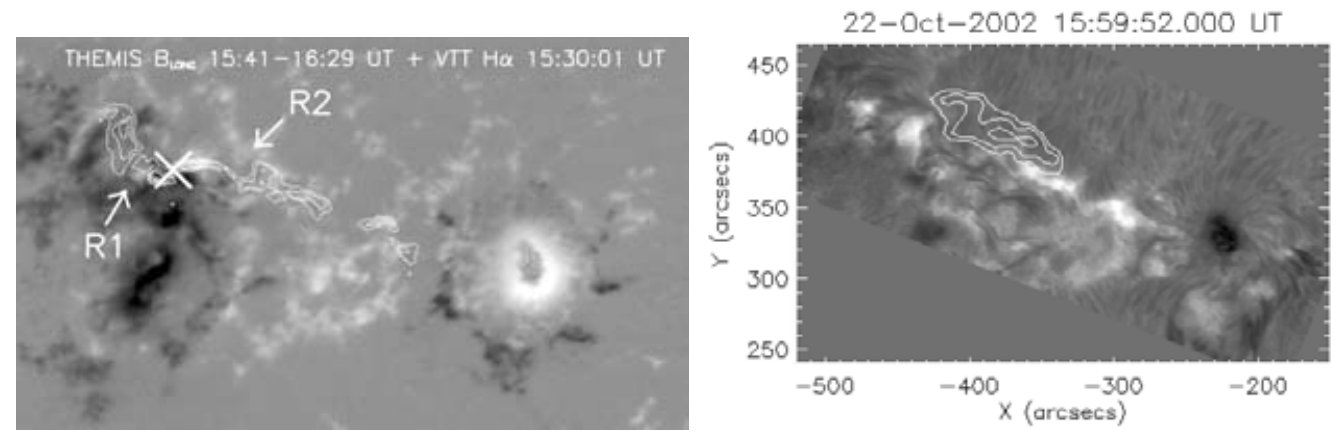

Figure 2. October 22, 2002, (left panel) magnetic field of the active region observed with THEMIS/MSDP, $\mathrm{H} \alpha$ ribbon contours are overlaid, (right panel) $\mathrm{H} \alpha$ two ribbon flare observed with the VTT/MSDP. Contours of RHESSI emission are overlaid.

\section{October 222002}

A compact twisted emerging flux (detected in the transverse magnetic field of Huairou) was responsible of the M1.9 flare, which remains a compact flare due to very tied overlaying loops (Figure 2). During this event we did not observe neither CME nor any other signatures of an eruption. This flare is a confined flare with no opening structures. RHESSI showed that even during the gradual phase of the M1.9 flare a non thermal component has to be taken into account to explain the observed spectra.

\section{Acknowledgements}

This work has been done in the frame of the ESMN (contract HPRN-CT-2002-00313).

\section{References}

Berlicki A., Schmieder B., Vilmer N., Aulanier G., DelZanna G. 2004 Astron. \& Astrophys., 423, 1129. 